\title{
Reconstructibility of time-invariant and periodic behavioral systems
}

\author{
J.C. Aleixo ${ }^{a *}$ and P. Rocha ${ }^{b}$ \\ ${ }^{a}$ Department of Mathematics, University of Beira Interior, Covilhã, Portugal; \\ ${ }^{b}$ Department of Electrical and Computer Engineering, Faculty of Engineering of the \\ University of Porto, Porto, Portugal
}

\begin{abstract}
In this paper the properties of behavioral reconstructibility and forward-observability for systems over the whole time axis $\mathbb{Z}$ are introduced. These properties are characterized in terms of appropriate rank conditions, for the time-invariant case. A comparison is made with the existing results in the behavioral setting as well as in the classical state space framework. In the particular case of a periodic system it is shown that there exists an equivalence between the reconstructibility of the periodic system and its associated lifted system, which is timeinvariant. Furthermore, we prove that, for a classical state space system, state reconstructibility is equivalent to behavioral reconstructibility, regardless of the time varying or time-invariant nature of the system. This allows deriving rank tests for the cases of time-invariant and of periodic systems, rediscovering the already known results for state reconstructibility from an alternative perspective. The obtained results contribute to establishing links between two different settings, thus providing a better insight into the considered systems properties.
\end{abstract}

Keywords: behaviors; state space systems; reconstructibility; lifted system.

\section{Introduction}

In the context of the behavioral approach, a dynamical system is characterized by the set of its admissible signals, called the system behavior. These signals correspond to the time evolution of the system variables, which have an "external" nature (in spite of not being divided into inputs and outputs). State variables are viewed as auxiliary variables that may, or not, be present in the system description. In this context one can still define several structural properties that were classically only considered for state space models.

In this paper we focus on the property of reconstructibility for linear discrete-time behavioral systems, considering both the time-invariant and the periodic cases. Behavioral observability has been defined in (Willems 1989, 1991, Polderman and Willems 1998), whereas behavioral reconstructibility has been introduced in (Valcher and Willems 1999a,b) for systems over $\mathbb{Z}_{+}$.

Given a linear behavioral system whose variable $w$ is partitioned into two sub-variables $w_{1}$ and $w_{2}, w_{2}$ is said to be observable from $w_{1}$ if whenever the trajectory $w_{1}$ is null overall in time, the same happens for the trajectory $w_{2}$. For systems defined on the half-line $\mathbb{Z}_{+}$, this can be viewed as the behavioral equivalent of the classical definition of state observability, according to which the knowledge of the (future) evolution of the inputs and outputs gives information about the initial state, and hence also gives information about the whole state trajectory from the initial instant on. However, this does not happen for systems defined over $\mathbb{Z}$, since the definition of behavioral observability has a global character, as it involves the values of the trajectories over the whole time axis, rather than over a half-line. Here we propose a generalization of the 
notion of reconstructibility introduced in (Valcher and Willems 1999a,b) for systems over $\mathbb{Z}_{+}$ to systems over $\mathbb{Z}$ that only considers half-line information. Very loosely speaking, in a linear behavioral system whose variable $w$ is partitioned into two sub-variables $w_{1}$ and $w_{2}, w_{2}$ is said to be reconstructible from $w_{1}$ if whenever the $w_{1}$ is null from some time instant on, the same happens with the trajectory $w_{2}$, but possibly with some time delay. When this delay is not present, we say that the system is forward observable. This corresponds to the usual notion of observability for state space systems.

Our purpose is to investigate the relationships among the different aforementioned notions. In order to give a better insight, we also relate the state space framework to the behavioral one. This is done not only for time-invariant, but also for periodic behavioral systems.

In Section 2 we introduce some preliminary notions and facts about behaviors. Section 3 is devoted to the definition of reconstructibility and forward-observability for systems over $\mathbb{Z}$. For time-invariant systems these properties are compared with other existing behavioral notions and are characterized in terms of the matrices that are used in the corresponding behavior description. Using the lifting formulation, a proof is presented that relates the reconstructibility of a periodic system and of its lifted associated system. Moreover, a rank characterization is derived. In Section 4 the classical notions of state reconstructibility and state observability are recalled and related to our behavioral definition of reconstructibility. Finally reconstructibility tests for time-invariant and periodic state space systems are derived using our behavioral results. Conclusions are presented in Section 5.

\section{Background}

In the behavioral framework, see (Willems 1989, 1991), a dynamical system $\Sigma$ is defined as a triple $\Sigma=(\mathbb{T}, \mathbb{W}, \mathfrak{B})$, where $\mathbb{T} \subseteq \mathbb{R}$ is the time set, $\mathbb{W}$ is the signal space and $\mathfrak{B} \subseteq \mathbb{W}^{\mathbb{T}}:=\{w: \mathbb{T} \rightarrow \mathbb{W}\}$ is the behavior. The behavior $\mathfrak{B}$ is what characterizes the phenomenon described by the system $\Sigma$, since it consists of all the signal evolutions (system trajectories) that are compatible with the laws of that phenomenon. Here we shall be concerned with the discrete-time case, more concretely, $\mathbb{T}=\mathbb{Z}$, assuming furthermore that the signal space is $\mathbb{W}=\mathbb{R}^{q}$, with $q \in \mathbb{N}$. Thus, $\mathbb{W}^{\mathbb{T}}=\left(\mathbb{R}^{q}\right)^{\mathbb{Z}}$, i.e., the system trajectories are $\mathbb{R}^{q}$-valued sequences over $\mathbb{Z}$.

In this section we recall some fundamentals of the behavioral approach.

Definition 2.1: (Willems 1989, 1991) A dynamical system $\Sigma=(\mathbb{T}, \mathbb{W}, \mathfrak{B})$ is said to be linear if $\mathbb{W}$ is a vector space (over a field $\mathbb{F}$ ) and $\mathfrak{B}$ is a linear subspace of $\mathbb{W}^{\mathbb{T}}$ (which is obviously a vector space when equipped with the usual operations of point-wise addition and multiplication by a scalar).

In order to introduce the notion of time-invariance it is essential to define first the time shift for signals $w$, defined over $\mathbb{Z}$.

We define the backward time shift as $\sigma:\left(\mathbb{R}^{q}\right)^{\mathbb{Z}} \rightarrow\left(\mathbb{R}^{q}\right)^{\mathbb{Z}}$, such that:

$$
(\sigma w)(k):=w(k+1)
$$

while the forward time shift $\sigma^{-1}:\left(\mathbb{R}^{q}\right)^{\mathbb{Z}} \rightarrow\left(\mathbb{R}^{q}\right)^{\mathbb{Z}}$ is such that:

$$
\left(\sigma^{-1} w\right)(k):=w(k-1) .
$$

Given a positive integer $L$, the composition of $L$ backward/forward shifts is denoted as $\sigma^{L} / \sigma^{-L}$. 
Definition 2.2: (Willems 1989, 1991) A dynamical system $\Sigma=\left(\mathbb{Z}, \mathbb{R}^{q}, \mathfrak{B}\right)$ is said to be timeinvariant if $\sigma(\mathfrak{B})=\mathfrak{B}$.

Thus, time-invariance is nothing else than the invariance of the set $\mathfrak{B}$ with respect to the time shift $\sigma$ and its inverse $\sigma^{-1}$.

Another important notion is the completeness of a behavior $\mathfrak{B}$, meaning, roughly speaking, that it is possible to check whether a trajectory $w \in\left(\mathbb{R}^{q}\right)^{\mathbb{Z}}$ belongs to $\mathfrak{B}$, by checking what happens in the set $\mathfrak{I}$ of finite intervals of $\mathbb{Z}$. Given $I \in \mathfrak{I}$, denote by $\left.\mathfrak{B}\right|_{I}$ the set of all the restrictions of the system trajectories to the time interval $I$, that is,

$$
\left.\mathfrak{B}\right|_{I}=\left\{\left.w\right|_{I}: w \in \mathfrak{B}\right\} \text {. }
$$

Definition 2.3: (Willems 1989, 1991) A dynamical system $\Sigma=(\mathbb{Z}, \mathbb{W}, \mathfrak{B})$ is said to be complete if

$$
\left(\forall I \in \mathfrak{I},\left.\left.\quad w\right|_{I} \in \mathfrak{B}\right|_{I}\right) \Leftrightarrow w \in \mathfrak{B}
$$

Interesting insights into this property and the connection between systems over $\mathbb{Z}$ and systems over $\mathbb{Z}_{+}$are given in (Lomadze et al. 1998).

A crucial issue is the representation of the behavior of a system by means of mathematical equations. It turns out that all the discrete-time dynamical systems that are linear, time-invariant and complete, allow a special type of mathematical description known as kernel representation, see (Willems 1989, 1991).

Theorem 2.4: (Willems 1989, 1991) Let $\Sigma=\left(\mathbb{Z}, \mathbb{R}^{q}, \mathfrak{B}\right)$ be a dynamical system. The following are equivalent:

i) $\Sigma$ is linear, time-invariant and complete;

ii) $\exists R\left(\xi, \xi^{-1}\right) \in \mathbb{R}^{\bullet \times q}\left[\xi, \xi^{-1}\right]$ such that

$$
\mathfrak{B}=\operatorname{ker} R\left(\sigma, \sigma^{-1}\right):=\left\{w \in\left(\mathbb{R}^{q}\right)^{\mathbb{Z}}: R\left(\sigma, \sigma^{-1}\right) w=0\right\}
$$

where $\mathbb{R}^{\bullet \times q}\left[\xi, \xi^{-1}\right]$ denotes the set of $\bullet \times q$ matrices with entries in $\mathbb{R}\left[\xi, \xi^{-1}\right]$, the ring of Laurent-polynomials in the indeterminate $\xi$.

Condition ii), in Theorem 2.4, means that there exists a certain Laurent-polynomial matrix

$$
R\left(\xi, \xi^{-1}\right)=R^{-M} \xi^{-M}+\cdots+R^{0}+\cdots+R^{N} \xi^{N},
$$

with $N, M \in \mathbb{Z}_{+}$, such that the trajectories $w \in \mathfrak{B}$ are the elements of $\left(\mathbb{R}^{q}\right)^{\mathbb{Z}}$ which constitute a solution of the linear constant coefficient matrix difference equation

$R^{-M} w(k-M)+\cdots+R^{-1} w(k-1)+R^{0} w(k)+R^{1} w(k+1)+\cdots+R^{N} w(k+N)=0, \forall k \in \mathbb{Z}$.

This matrix $R\left(\xi, \xi^{-1}\right)$ is called a kernel representation $(K R)$ matrix of $\mathfrak{B}$. A behavior that allows a KR is called kernel behavior.

While the behavior $\mathfrak{B}$ of a time-invariant system over $\mathbb{Z}$ is characterized by its invariance under the time shift (and its inverse), $P$-periodic behaviors are required to be invariant only with respect to the $P^{\text {th }}$ power of the shift, and of its inverse. 
Definition 2.5: (Kuijper and Willems 1997) A system $\Sigma$ is said to be $P$-periodic, with $P \in \mathbb{N}$, if its behavior $\mathfrak{B}$ satisfies

$$
\sigma^{P} \mathfrak{B}=\mathfrak{B}
$$

but not $\sigma^{Q} \mathfrak{B}=\mathfrak{B}$, for any $Q \in \mathbb{N}$ smaller than $P$.

Remark 1: Note that, regarding time-invariance as 1-periodicity, Definition 2.5 yields the usual definition of time-invariant systems.

According to (Kuijper and Willems 1997), a behavior $\mathfrak{B}$ is a $\sigma^{P}$-invariant linear closed subspace of $\left(\mathbb{R}^{q}\right)^{\mathbb{Z}}$ (in the topology of point-wise convergence) if and only if it has a representation of the type

$$
\left(R_{t}\left(\sigma, \sigma^{-1}\right) w\right)(t+P k)=0, t=0, \ldots, P-1, k \in \mathbb{Z}
$$

where, for each time instant $t=0, \ldots, P-1$,

$$
R_{t}\left(\xi, \xi^{-1}\right) \in \mathbb{R}^{g_{t} \times q}\left[\xi, \xi^{-1}\right]
$$

is a Laurent polynomial matrix in the indeterminate $\xi$. Note that the Laurent-polynomial matrices $R_{t}$ need not have the same number of rows (in fact we could even have some $g_{t}$ equal to zero, meaning that the corresponding matrix $R_{t}$ would be void and no restrictions were imposed at the time instants $P k+t$ ). Equation (1) can also be written as

$$
\left(R\left(\sigma, \sigma^{-1}\right) w\right)(P k)=0, \quad k \in \mathbb{Z}
$$

where

$$
R\left(\xi, \xi^{-1}\right):=\left[\begin{array}{c}
R_{0}\left(\xi, \xi^{-1}\right) \\
\xi R_{1}\left(\xi, \xi^{-1}\right) \\
\vdots \\
\xi^{P-1} R_{P-1}\left(\xi, \xi^{-1}\right)
\end{array}\right] \in \mathbb{R}^{g \times q}\left[\xi, \xi^{-1}\right]
$$

with $g:=\sum_{t=0}^{P-1} g_{t}$. In the $P$-periodic case, with $P \geqslant 2$, with some abuse of language, we refer to $(2)$ as a $P$-periodic kernel representation $(P-P K R)$. Clearly, in the time-invariant case, where $P=1,(2)$ particularizes to

$$
\left(R\left(\sigma, \sigma^{-1}\right) w\right)(k)=0, \quad k \in \mathbb{Z}
$$

Consider now that the system variable $w$ is partitioned as $\left(w_{1}, w_{2}\right)$. In this case, the corresponding behavior description (2) can be written as

$$
\left(R_{2}\left(\sigma, \sigma^{-1}\right) w_{2}\right)(P k)=\left(R_{1}\left(\sigma, \sigma^{-1}\right) w_{1}\right)(P k), \quad k \in \mathbb{Z},
$$

where the Laurent-polynomial matrices $R_{i}\left(\xi, \xi^{-1}\right) \in \mathbb{R}^{g \times q_{i}}\left[\xi, \xi^{-1}\right], g:=\sum_{t=0}^{P-1} g_{t}, i=1,2$, are obtained by means of a suitable partition (and, if necessary, rearrangement) of the columns of $R$.

By decomposing matrices $R_{2}$ and $R_{1}$ as, see (Aleixo et al. 2007),

$$
R_{i}\left(\xi, \xi^{-1}\right)=R_{i}^{L}\left(\xi^{P}, \xi^{-P}\right) \Omega_{P, q_{i}}(\xi), \quad i=1,2
$$


where

$$
\Omega_{P, q_{i}}(\xi):=\left[\begin{array}{llll}
I_{q_{i}} & \xi I_{q_{i}} & \cdots & \xi^{P-1} I_{q_{i}}
\end{array}\right]^{T},
$$

we may write down relation (3) as

$$
\left(R_{2}^{L}\left(\sigma^{P}, \sigma^{-P}\right) \Omega_{P, q_{2}}(\sigma) w_{2}\right)(P k)=\left(R_{1}^{L}\left(\sigma^{P}, \sigma^{-P}\right) \Omega_{P, q_{1}}(\sigma) w_{1}\right)(P k), k \in \mathbb{Z} .
$$

Defining the lifted trajectories

$$
\left(L w_{i}\right)(k)=\left[\begin{array}{c}
w_{i}(P k) \\
\vdots \\
w_{i}(P k+P-1)
\end{array}\right], \quad i=1,2
$$

see (Urbano 1987, Hernández and Urbano 1987, Khargonekar et al. 1985, Kuijper and Willems 1997, Aleixo et al. 2007), and noting that $L \sigma^{P}=\sigma L$, (4) may be written as

$$
\left(R_{2}^{L}\left(\sigma, \sigma^{-1}\right)\left(L w_{2}\right)\right)(k)=\left(R_{1}^{L}\left(\sigma, \sigma^{-1}\right)\left(L w_{1}\right)\right)(k), \quad k \in \mathbb{Z} .
$$

The behavior $L \mathfrak{B}$, defined by $\{L w, w \in \mathfrak{B}\}$, called the lifted behavior, is equal to the set of trajectories

$$
\left\{\left(L w_{1}, L w_{2}\right) \in\left(\mathbb{R}^{P q_{1}}\right)^{\mathbb{Z}} \times\left(\mathbb{R}^{P q_{2}}\right)^{\mathbb{Z}} \mid(5) \text { holds }\right\}
$$

that is,

$$
L \mathfrak{B}=\operatorname{ker} \widetilde{R}^{L}\left(\sigma, \sigma^{-1}\right)
$$

where $\widetilde{R}^{L}\left(\xi, \xi^{-1}\right):=\left[R_{2}^{L}\left(\xi, \xi^{-1}\right) \quad R_{1}^{L}\left(\xi, \xi^{-1}\right)\right]$. In particular, this implies that $L \mathfrak{B}$ is timeinvariant.

A well-known technique in the study of periodic systems consists in relating the structural properties of these systems with the properties of corresponding time-invariant formulations. In this paper we shall make use of the (time-invariant) lifted behavior in order to obtain a characterization of reconstructibility for the periodic case.

\section{Behavioral reconstructibility}

Similar to controllability and reachability, the properties of reconstructibility and observability also play a central role in systems theory. As in the classical case, for behavioral systems these properties are related with the possibility of obtaining information about some components of the system variable, which cannot be directly measured, based on the knowledge of the other components, which are assumed to be available for measurement.

\subsection{Definition}

Definition 3.1 Behavioral reconstructibility: Let $\mathfrak{B} \subset\left(\mathbb{R}^{q}\right)^{\mathbb{Z}} \simeq\left(\mathbb{R}^{q_{1}} \times \mathbb{R}^{q_{2}}\right)^{\mathbb{Z}}$ be a behavior whose system variable $w$ is partitioned as $w=\left(w_{1}, w_{2}\right)$. Given $\delta \geqslant 0$, we say that $w_{2}$ is $\delta$ - 
reconstructible from $w_{1}$ if

$$
\left\{\left.w_{1}\right|_{\left[k_{0},+\infty\right)} \equiv 0\right\} \Rightarrow\left\{\left.w_{2}\right|_{\left[k_{0}+\delta,+\infty\right)} \equiv 0\right\}, \quad \forall k_{0} \in \mathbb{Z}
$$

Moreover, $w_{2}$ is said to be reconstructible from $w_{1}$ if it is $\delta$-reconstructible from $w_{1}$ for some $\delta \geqslant 0$. In this case $\mathfrak{B}$ is also said to be reconstructible with respect to $w_{2}$. In particular, $w_{2}$ is said to be forward-observable from $w_{1}$ if it is 0-reconstructible from $w_{1}$, i.e., if

$$
\left\{\left.w_{1}\right|_{\left[k_{0},+\infty\right)} \equiv 0\right\} \Rightarrow\left\{\left.w_{2}\right|_{\left[k_{0},+\infty\right)} \equiv 0\right\}, \quad \forall k_{0} \in \mathbb{Z} .
$$

In this case $\mathfrak{B}$ is said to be forward-observable with respect to $w_{2}$.

Remark 2: In this definition and throughout the entire paper, the interval notation $[a, b]$ is to be understood as $[a, b] \cap \mathbb{Z}$.

Example 3.2 Consider a time-invariant system $\Sigma=\left(\mathbb{Z}, \mathbb{R}^{2}, \mathfrak{B}\right)$ with variables $\left(w_{1}, w_{2}\right)$, whose behavior $\mathfrak{B}$ is described by

$$
\sigma w_{2}=w_{1}
$$

i.e.,

$$
w_{2}(k)=w_{1}(k-1), \quad \forall k \in \mathbb{Z} .
$$

Clearly $w_{2}$ is 1-reconstructible from $w_{1}$, since

$$
w_{1}(k)=0, \quad k \geqslant k_{0}
$$

implies

$$
w_{2}(k)=w_{1}(k-1)=0, \quad k \geqslant k_{0}+1 .
$$

It is also simple to see that $w_{2}$ is not forward-observable from $w_{1}$. Indeed, if $w_{1}(-1)=1$ and $w_{1}(k)=0$ for $k \neq-1$, we have that $w_{2}(0)=1$ and $w_{2}(k)=0$ for $k \neq 0$. Thus

$$
\left.w_{1}\right|_{[0,+\infty)}=0, \text { but }\left.\quad w_{2}\right|_{[0,+\infty)} \neq 0 .
$$

However, $w_{1}$ is forward-observable from $w_{2}$, as can easily be ascertained.

\subsection{The time-invariant case}

Observe that, in case of time-invariance, the $\delta$-reconstructibility condition (6) in Definition 3.1 can be replaced by

$$
\left\{\left.w_{1}\right|_{[0,+\infty)} \equiv 0\right\} \Rightarrow\left\{\left.w_{2}\right|_{[\delta,+\infty)} \equiv 0\right\}
$$

whereas the forward-observability condition (7) can be replaced by

$$
\left\{\left.w_{1}\right|_{[0,+\infty)} \equiv 0\right\} \Rightarrow\left\{\left.w_{2}\right|_{[0,+\infty)} \equiv 0\right\}
$$


This agrees with the definitions of reconstructibility and observability given in (Valcher and Willems 1999b), for discrete-time systems over $\mathbb{Z}_{+}$. However, it does not agree with the definition of observability given in (Willems 1989, 1991), according to which $w_{2}$ is said to be observable from $w_{1}$ if

$$
\left\{w_{1}(k)=0, \forall k \in \mathbb{Z}\right\} \Rightarrow\left\{w_{2}(k)=0, \forall k \in \mathbb{Z}\right\}
$$

In the sequel, to avoid confusion we refer to this latter property as Willems-observability.

Proposition 3.3: Let $\mathfrak{B} \subset\left(\mathbb{R}^{q}\right)^{\mathbb{Z}}$ be a time-invariant behavior whose system variable $w$ is partitioned as $w=\left(w_{1}, w_{2}\right)$. Then $w_{2}$ is Willems-observable from $w_{1}$ if it is reconstructible from $w_{1}$.

\section{Proof}

Assume that $w_{2}$ is $\delta$-reconstructible from $w_{1}$, for some $\delta \geqslant 0$. Consider a trajectory $\left(w_{1}^{\prime}, w_{2}^{\prime}\right) \in$ $\mathfrak{B}$ with $\left.w_{1}^{\prime}\right|_{\mathbb{Z}}=0$. In particular,

$$
\left.w_{1}^{\prime}\right|_{\left[k_{0},+\infty\right)} \equiv 0, \quad \forall k_{0} \in \mathbb{Z}
$$

and therefore

$$
\left.w_{2}^{\prime}\right|_{\left[k_{0}+\delta,+\infty\right)} \equiv 0, \quad \forall k_{0} \in \mathbb{Z}
$$

This clearly implies that $\left.w_{2}^{\prime}\right|_{\mathbb{Z}} \equiv 0$, allowing to conclude that reconstructibility implies Willemsobservability.

As we shall see in the sequel, the reciprocal of this result is also valid. For that purpose, we first characterize reconstructibility and forward-observability by means of rank conditions.

Theorem 3.4: Consider the time-invariant dynamical system $\Sigma=\left(\mathbb{Z}, \mathbb{R}^{q_{1}+q_{2}}, \mathfrak{B}\right)$ described by

$$
\mathfrak{B}:=\left\{\left(w_{1}, w_{2}\right) \in\left(\mathbb{R}^{q_{1}+q_{2}}\right)^{\mathbb{Z}} \mid\left(R_{2}\left(\sigma, \sigma^{-1}\right) w_{2}\right)(k)=\left(R_{1}\left(\sigma, \sigma^{-1}\right) w_{1}\right)(k), k \in \mathbb{Z}\right\},
$$

with $R_{i}\left(\xi, \xi^{-1}\right) \in \mathbb{R}^{g \times q_{i}}\left[\xi, \xi^{-1}\right], i=1,2$. Then,

i) $w_{2}$ is reconstructible from $w_{1}$ if and only if

$$
\operatorname{rank} R_{2}\left(\lambda, \lambda^{-1}\right)=q_{2}, \quad \forall \lambda \in \mathbb{C} \backslash\{0\} ;
$$

ii) $w_{2}$ is forward-observable from $w_{1}$ if and only if there exist $\widetilde{R}_{2}(\xi) \in \mathbb{R}^{g \times q_{2}}[\xi]$ and $\widetilde{R}_{1}(\xi) \in$ $\mathbb{R}^{g \times q_{1}}[\xi]$ such that $\mathfrak{B}$ is described by $\widetilde{R}_{2}(\sigma) w_{2}=\widetilde{R}_{1}(\sigma) w_{1}$, with

$$
\operatorname{rank} \widetilde{R}_{2}(\lambda)=q_{2}, \quad \forall \lambda \in \mathbb{C} .
$$

\section{Proof}

i) Assume that (10) holds. Then, there exists a matrix $U\left(\xi, \xi^{-1}\right) \in \mathbb{R}^{g \times g}\left[\xi, \xi^{-1}\right]$, which is 
unimodular $^{1}$ over $\mathbb{R}\left[\xi, \xi^{-1}\right]$, such that (Polderman and Willems 1998),

$$
U\left(\xi, \xi^{-1}\right) R_{2}\left(\xi, \xi^{-1}\right)=\left[\begin{array}{c}
I_{q_{2}} \\
0
\end{array}\right]
$$

Thus (leaving out $\sigma$ and $\sigma^{-1}$ in the notation, for simplicity),

$$
\begin{aligned}
R_{2} w_{2}=R_{1} w_{1} & \Leftrightarrow U R_{2} w_{2}=U R_{1} w_{1} \Leftrightarrow\left[\begin{array}{c}
I_{q_{2}} \\
0
\end{array}\right] w_{2}=\left[\begin{array}{c}
R_{1}^{1} \\
R_{1}^{2}
\end{array}\right] w_{1} \\
& \Leftrightarrow R_{1}^{2} w_{1}=0 \quad \text { and } \quad w_{2}=R_{1}^{1} w_{1}
\end{aligned}
$$

with $U R_{1}$ conformably partitioned as

$$
U\left(\xi, \xi^{-1}\right) R_{1}\left(\xi, \xi^{-1}\right)=\left[\begin{array}{l}
R_{1}^{1}\left(\xi, \xi^{-1}\right) \\
R_{1}^{2}\left(\xi, \xi^{-1}\right)
\end{array}\right] .
$$

Let

$$
R_{1}^{1}\left(\xi, \xi^{-1}\right)=R_{1}^{1^{-M}} \xi^{-M}+\cdots+R_{1}^{1^{0}}+\cdots+R_{1}^{1^{N}} \xi^{N}
$$

with $N, M \in \mathbb{Z}_{+}$. Applying $\sigma^{M}$ to both sides of the equality $w_{2}=R_{1}^{1} w_{1}$, we obtain

$$
\left(\sigma^{M} w_{2}\right)(k)=\left(\widetilde{R}_{1}^{1}(\sigma) w_{1}\right)(k), \quad k \in \mathbb{Z}
$$

allowing us to conclude that

$$
\left\{\left.w_{1}\right|_{\left[k_{0},+\infty\right)}=0\right\} \Rightarrow\left\{\left.w_{2}\right|_{\left[k_{0}+M,+\infty\right)}=0\right\}
$$

i.e., $w_{2}$ is $M$-reconstructible, and hence reconstructible, from $w_{1}$.

Suppose now that (10) does not hold. Then, there exists a trajectory $w_{2}^{*} \in \operatorname{ker} R_{2}\left(\sigma, \sigma^{-1}\right)$, which is non-zero (Polderman and Willems 1998). This trajectory is such that

$$
w^{*}=\left(w_{1}^{*} \equiv 0, w_{2}^{*}\right) \in \mathfrak{B} .
$$

If $w_{2}$ were reconstructible from $w_{1}$, this would imply that

$$
\left.w_{2}^{*}\right|_{\left[k^{*},+\infty\right)} \equiv 0, \quad \forall k^{*} \in \mathbb{Z}
$$

and, consequently, $w_{2}^{*}$ would be null in the whole time-axis $\mathbb{Z}$, which is a contradiction. Therefore, if the rank condition (10) does not hold, $w_{2}$ is not reconstructible from $w_{1}$, or, in other words, the reconstructibility of $w_{2}$ from $w_{1}$ implies that (10) holds;

ii) Suppose now that there exist $\widetilde{R}_{2}$ and $\widetilde{R}_{1}$ such that $\mathfrak{B}$ is described by

$$
\left(\widetilde{R}_{2}(\sigma) w_{2}\right)(k)=\left(\widetilde{R}_{1}(\sigma) w_{1}\right)(k), \quad k \in \mathbb{Z},
$$

\footnotetext{
${ }^{1}$ Given a ring $\mathcal{R}$, a square matrix $M \in \mathcal{R}^{g \times g}$ is said to be unimodular over $\mathcal{R}$ if it has an inverse in $\mathcal{R}^{g \times g}$.
} 
with $\widetilde{R}_{2}(\xi)$ satisfying (11). Then, there exists an unimodular matrix (over $\left.\mathbb{R}[\xi]\right) U(\xi)$ such that (Polderman and Willems 1998),

$$
U(\xi) \widetilde{R}_{2}(\xi)=\left[\begin{array}{c}
I_{q_{2}} \\
0
\end{array}\right]
$$

Thus,

$$
\begin{aligned}
\widetilde{R}_{2} w_{2}=\widetilde{R}_{1} w_{1} & \Leftrightarrow U \widetilde{R}_{2} w_{2}=U \widetilde{R}_{1} w_{1} \Leftrightarrow\left[\begin{array}{c}
I_{q_{2}} \\
0
\end{array}\right] w_{2}=\left[\begin{array}{c}
\widetilde{R}_{1}^{1} \\
\widetilde{R}_{1}^{2}
\end{array}\right] w_{1} \\
& \Leftrightarrow \widetilde{R}_{1}^{2} w_{1}=0 \text { and } w_{2}=\widetilde{R}_{1}^{1} w_{1},
\end{aligned}
$$

with $U \widetilde{R}_{1}$ conformably partitioned as

$$
U(\xi) \widetilde{R}_{1}(\xi)=\left[\begin{array}{c}
\widetilde{R}_{1}^{1}(\xi) \\
\widetilde{R}_{1}^{2}(\xi)
\end{array}\right] .
$$

Thus, if $w_{1}(k)=0$ for $k \in\left[k_{0},+\infty\right)$, then

$$
\left(\widetilde{R}_{1}^{1}(\sigma) w_{1}\right)(k)=0, \text { for } k \in\left[k_{0},+\infty\right)
$$

and hence

$$
w_{2}(k)=0, \quad \text { for } k \in\left[k_{0},+\infty\right),
$$

which allows us to conclude that $w_{2}$ is forward-observable from $w_{1}$.

Assume now that $w_{2}$ is forward-observable from $w_{1}$ and let

$$
\left(\widehat{R}_{2}(\sigma) w_{2}\right)(k)=\left(\widehat{R}_{1}(\sigma) w_{1}\right)(k), \quad k \in \mathbb{Z},
$$

be a representation of $\mathfrak{B}$. Consider a trajectory $\left(w_{1}, w_{2}\right) \in \mathfrak{B}$ such that $w_{1} \equiv 0$. Then, by the forward-observability of $\mathfrak{B}$, this implies that

$$
\forall k_{0} \in \mathbb{Z}, w_{2}(k)=0, \quad k \geqslant k_{0},
$$

or, in other words, $w_{2} \equiv 0$. This means that $\operatorname{ker} \widehat{R}_{2}(\sigma)=\{0\}$, which is equivalent to say, see (Willems 1991), that

$$
\operatorname{rank} \widehat{R}_{2}(\lambda)=\text { const, } \quad \forall \lambda \in \mathbb{C} \backslash\{0\} .
$$

Note that this also follows immediately from the previous item and from noticing that forward-observability implies reconstructibility.

Let now $U(\xi)$ and $V(\xi)$ be unimodular matrices (over $\mathbb{R}[\xi]$ ) that bring $\widehat{R}_{2}$ into its Smith 
form, i.e.,

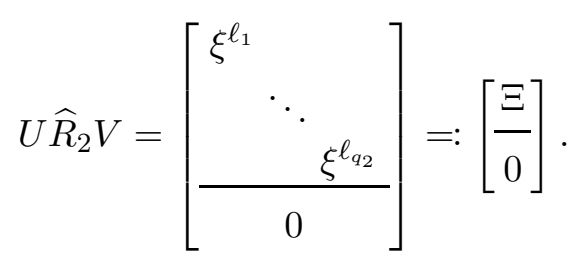

Then,

$$
\widehat{R}_{2} w_{2}=\widehat{R}_{1} w_{1} \Leftrightarrow U \widehat{R}_{2} w_{2}=U \widehat{R}_{1} w_{1} \Leftrightarrow\left[\frac{\Xi}{0}\right] V^{-1} w_{2}=\left[\begin{array}{c}
\widehat{R}_{1}^{1} \\
\widehat{R}_{1}^{2}
\end{array}\right] w_{1},
$$

which is equivalent to

$$
\widehat{R}_{1}^{2} w_{1}=0 \quad \text { and } \quad w_{2}=V \Xi^{-1} \widehat{R}_{1}^{1} w_{1}
$$

with $U \widehat{R}_{1}$ conformably partitioned as

$$
U(\xi) \widehat{R}_{1}(\xi)=\left[\begin{array}{c}
\widehat{R}_{1}^{1}(\xi) \\
\widehat{R}_{1}^{2}(\xi)
\end{array}\right]
$$

Thus, $\mathfrak{B}$ is also described by

$$
\left(\widetilde{R}_{2}(\sigma) w_{2}\right)(k)=\left(\widetilde{R}_{1}(\sigma) w_{1}\right)(k),
$$

with $\widetilde{R}_{2}, \widetilde{R}_{1}$ defined by

$$
\begin{aligned}
& \widetilde{R}_{2}(\xi):=\left[\begin{array}{c}
I_{q_{2}} \\
0
\end{array}\right] \\
& \widetilde{R}_{1}(\xi)=\left[\begin{array}{c}
\widetilde{R}_{1}^{1}(\xi) \\
\widetilde{R}_{1}^{2}(\xi)
\end{array}\right]:=\left[\begin{array}{c}
V(\xi) \Xi^{-1}(\xi) \widehat{R}_{1}^{1}(\xi) \\
\widehat{R}_{1}^{2}(\xi)
\end{array}\right] .
\end{aligned}
$$

Now, using the forward-observability property, it is clear that the matrix $\widetilde{R}_{1}^{1}:=V \Xi^{-1} \widehat{R}_{1}^{1}$ cannot have terms in $\xi^{-1}$. Moreover, $\widetilde{R}_{2}(\xi)$ has constant column rank over $\mathbb{C}$. This shows that there exists a representation of $\mathfrak{B}$ with the desired properties.

The proof is thus completed.

The Willems-observability condition (9) for behaviors over $\mathbb{Z}$ described by

$$
R_{2}\left(\sigma, \sigma^{-1}\right) w_{2}=R_{1}\left(\sigma, \sigma^{-1}\right) w_{1}
$$

is equivalent to the rank condition

$$
\operatorname{rank} R_{2}\left(\lambda, \lambda^{-1}\right)=q_{2}, \quad \forall \lambda \in \mathbb{C} \backslash\{0\}
$$

see (Willems 1991). This coincides with the condition (10) in Theorem 3.4-i), thus leading to the conclusion that Willems-observability is equivalent to our notion of reconstructibility, rather than to forward-observability. 
Proposition 3.5: Let $\mathfrak{B} \subset\left(\mathbb{R}^{q}\right)^{\mathbb{Z}}$ be a time-invariant behavior whose system variable $w$ is partitioned as $w=\left(w_{1}, w_{2}\right)$. Then $w_{2}$ is Willems-observable from $w_{1}$ if and only if it is reconstructible from $w_{1}$.

Note that the definition of observability, given in (Valcher and Willems 1999b), for systems over $\mathbb{Z}_{+}$can be regarded as an adaptation of Willems's definition (9), since it means that if $w_{1}$ is the null trajectory (i.e., is zero over the time-axis $\mathbb{Z}_{+}$), then the same happens for $w_{2}$. However, that notion can also be seen as an adaptation of our definition of forward-observability.

The situation is summarized in the following table containing, in bold, the conditions that define each of the properties

\begin{tabular}{lcc}
\hline & \multicolumn{2}{c}{$\mathbb{T}$} \\
\cline { 2 - 3 } Property & $\mathbb{Z}$ & $\mathbb{Z}_{+}$ \\
\hline Forward-observability & $(\mathbf{C} 1)$ & $(\mathbf{C} 1)$ \\
Willems-observability & $(\mathbf{C} 2)$ & $(\mathbf{C} 1)$ \\
Reconstructibility & $(\mathbf{C} 3) \Leftrightarrow(\mathrm{C} 2)$ & $(\mathbf{C} 3)$ \\
\hline
\end{tabular}

The conditions (C1), (C2) and (C3) are as follows:

(C1) $\left.w_{1}\right|_{\mathbb{Z}_{+}}=\left.0 \Rightarrow w_{2}\right|_{\mathbb{Z}_{+}}=0$;

(C2) $\left.w_{1}\right|_{\mathbb{Z}}=\left.0 \Rightarrow w_{2}\right|_{\mathbb{Z}}=0$;

(C3) $\exists \delta \geqslant 0$ s.t. $\left.w_{1}\right|_{\mathbb{Z}_{+}}=\left.0 \Rightarrow w_{2}\right|_{[\delta,+\infty)}=0$.

\subsection{The periodic case}

The relationship between the reconstructibility of a periodic behavior and of its lifted counterpart is given by the following result.

Theorem 3.6: Let $\Sigma=\left(\mathbb{Z}, \mathbb{R}^{q_{1}} \times \mathbb{R}^{q_{2}}, \mathfrak{B}\right)$ be a P-periodic system whose system variable $w$ is partitioned as $w=\left(w_{1}, w_{2}\right)$, described by $(3)$, and $\Sigma^{L}$ its associated lifted system ${ }^{2}$. Then the following are equivalent:

i) $\mathfrak{B}$ is reconstructible with respect to $w_{2}$;

ii) $L \mathfrak{B}$ is reconstructible with respect to $L w_{2}$;

iii) $\operatorname{rank} R_{2}^{L}\left(\lambda, \lambda^{-1}\right)=P q_{2}, \quad \forall \lambda \in \mathbb{C} \backslash\{0\}$.

Proof The equivalence between conditions ii) and iii) follows immediately from Theorem 3.4 and from the fact that $L \mathfrak{B}$ is a time-invariant behavior. Now, we prove that conditions i) and ii) are equivalent. Assume that $\mathfrak{B}$ is reconstructible with respect to $w_{2}$. Then, $w_{2}$ is $\delta$-reconstructible from $w_{1}$, for some $\delta \geqslant 0$, i.e., that

$$
\left\{\left.w_{1}\right|_{\left[k_{0},+\infty\right)} \equiv 0\right\} \Rightarrow\left\{\left.w_{2}\right|_{\left[k_{0}+\delta,+\infty\right)} \equiv 0\right\}, \forall k_{0} \in \mathbb{Z}
$$

Consider $\widetilde{w}_{1}, \widetilde{w}_{2} \in L \mathfrak{B}$ and $\widetilde{k}_{0} \in \mathbb{Z}$. Let $w_{1}, w_{2} \in \mathfrak{B}$ be such that $L w_{i}=\widetilde{w}_{i}, i=1,2$. Define

${ }^{2}$ Where $\Sigma^{L}=\left(\mathbb{Z}, \mathbb{R}^{P q_{1}} \times \mathbb{R}^{P q_{2}}, L \mathfrak{B}\right)$. 
$k_{0}:=P \widetilde{k}_{0}$ and take $\widetilde{\delta}=\left\lceil\frac{\delta}{P}\right\rceil^{3}$. Then, $\forall \widetilde{k}_{0} \in \mathbb{Z}$,

$$
\begin{aligned}
& \left\{\left.\widetilde{w}_{1}\right|_{\left[\widetilde{k}_{0},+\infty\right)} \equiv 0\right\} \Leftrightarrow\left\{\left[\begin{array}{c}
w_{1}(P k) \\
\vdots \\
w_{1}(P k+P-1)
\end{array}\right]=0, \forall k \in\left[\widetilde{k}_{0},+\infty\right)\right\} \\
& \Leftrightarrow\left\{w_{1}(\ell)=0, \quad \forall \ell \in\left[P \widetilde{k}_{0},+\infty\right)\right\} \Leftrightarrow\left\{w_{1}(\ell)=0, \quad \forall \ell \in\left[k_{0},+\infty\right)\right\} \\
& \stackrel{4}{\Rightarrow}\left\{w_{2}(\ell)=0, \quad \forall \ell \in\left[k_{0}+\delta,+\infty\right)\right\} \Leftrightarrow\left\{\left[\begin{array}{c}
w_{2}(P k) \\
\vdots \\
w_{2}(P k+P-1)
\end{array}\right]=0, \forall k \geqslant \frac{k_{0}+\delta}{P}\right\} \\
& \Leftrightarrow\left\{\left[\begin{array}{c}
w_{2}(P k) \\
\vdots \\
w_{2}(P k+P-1)
\end{array}\right]=0, \forall k \in\left[\widetilde{k}_{0}+\widetilde{\delta},+\infty\right)\right\} \Leftrightarrow\left\{\left.\widetilde{w}_{2}\right|_{\left[\widetilde{k}_{0}+\widetilde{\delta},+\infty\right)} \equiv 0\right\},
\end{aligned}
$$

showing that $L w_{2}$ is $\widetilde{\delta}$-reconstructible from $L w_{1}$.

Assume now that $L \mathfrak{B}$ is reconstructible with respect to $L w_{2}$. Then, $L w_{2}$ is $\widetilde{\delta}$-reconstructible from $L w_{1}$, for some $\widetilde{\delta} \geqslant 0$. Let $w_{1}, w_{2} \in \mathfrak{B}$ and $k_{0} \in \mathbb{Z}$. Let further $\widetilde{w}_{1}, \widetilde{w}_{2} \in L \mathfrak{B}$ such that $L w_{i}=\widetilde{w}_{i}, i=1,2$.

Then,

$$
\begin{aligned}
& \left\{w_{1}(k)=0, \quad \forall k \in\left[k_{0},+\infty\right)\right\} \Leftrightarrow\left\{\left[\begin{array}{c}
w_{1}(P \ell) \\
\vdots \\
w_{1}(P \ell+P-1)
\end{array}\right]=0, \quad \forall \ell \geqslant \frac{k_{0}}{P}\right\} \\
& \Leftrightarrow\left\{\widetilde{w}_{1}(\ell)=0, \quad \forall \ell \geqslant\left\lceil\frac{k_{0}}{P}\right\rceil\right\} \stackrel{5}{\Rightarrow}\left\{\widetilde{w}_{2}(\ell)=0, \quad \forall \ell \geqslant\left\lceil\frac{k_{0}}{P}\right\rceil+\widetilde{\delta}\right\} \\
& \Leftrightarrow\left\{\left[\begin{array}{c}
w_{2}(P \ell) \\
\vdots \\
w_{2}(P \ell+P-1)
\end{array}\right]=0, \forall \ell \geqslant\left\lceil\frac{k_{0}}{P}\right\rceil+\widetilde{\delta}\right\} \Leftrightarrow\left\{w_{2}(k)=0, \forall k \geqslant k_{0}+P \widetilde{\delta}\right\} .
\end{aligned}
$$

This shows that $w_{2}$ is $\delta$-reconstructible from $w_{1}$ with $\delta=P \widetilde{\delta}$.

\footnotetext{
${ }^{3}\lceil\cdot\rceil$ represents the ceiling function, i.e., the integer round-up defined as $\lceil x\rceil=\min \{m \in \mathbb{Z}: m \geqslant x\}$.

${ }^{4}$ By the $\delta$-reconstructibility of $w_{2}$ from $w_{1}$.

${ }^{5}$ By the $\widetilde{\delta}$-reconstructibility of $L w_{2}$ from $L w_{1}$.
} 
The classical state space approach to linear, time-varying, systems takes as its starting point a description $\Sigma_{s}=(A(\cdot), B(\cdot), C(\cdot), D(\cdot))$ of the form

$$
\left\{\begin{array}{rl}
(\sigma x)(k) & =A(k) x(k)+B(k) u(k) \\
y(k) & =C(k) x(k)+D(k) u(k)
\end{array} \quad k \in \mathbb{Z},\right.
$$

where the matrices $A(k) \in \mathbb{R}^{n \times n}, B(k) \in \mathbb{R}^{n \times m}, C(k) \in \mathbb{R}^{p \times n}$ and $D(k) \in \mathbb{R}^{p \times m}$ depend on $k$, $x$ is the state variable and $u$ and $y$ are the input and output, respectively.

In this section we first recall the classical notions of state reconstructibility and state observability, and then view a state space system from the behavioral standpoint in order to compare classical and behavioral properties. This allows us to rediscover, from another perspective, already known results on the characterization of reconstructibility of state space systems.

\subsection{Classical notions}

The structural properties of state reconstructibility and state observability for time-varying systems are introduced following the spirit of the well-known versions for time-invariant systems, yielding more general definitions that also apply to the time-invariant case.

Definition 4.1 State reconstructibility :

i) A state $x_{1} \in \mathbb{R}^{n}$ is called unreconstructible at time $k_{1}$ if for all $k_{0} \leqslant k_{1}$, there exists $x_{0}=x\left(k_{0}\right) \in \mathbb{R}^{n}$ such that

$$
y(k)=C(k) \phi_{A}\left(k, k_{0}\right) x_{0}=0, \quad k \in\left[k_{0}, k_{1}-1\right],
$$

with $x_{1}=x\left(k_{1}\right)$;

ii) The system (13) is called completely state reconstructible at time $k_{1}$ if the only state $x_{1}$ that is unreconstructible at time $k_{1}$ is the zero state, i.e., $x_{1}=0 \in \mathbb{R}^{n}$. If this happens for all $k_{1} \in \mathbb{Z},(13)$ is simply called completely state reconstructible.

Definition 4.2 State observability :

i) A state $x_{0} \in \mathbb{R}^{n}$ is called unobservable (at time $k_{0}$ ) if for all $k_{1} \geqslant k_{0}$

$$
y(k)=C(k) \phi_{A}\left(k, k_{0}\right) x_{0}=0, \quad k \in\left[k_{0}, k_{1}-1\right]
$$

i.e., if the zero input response of the system is zero for every $k \geqslant k_{0}$;

ii) The system (13) is called completely state observable at time $k_{0}$ if the only state $x_{0}$ that is unobservable is the zero state, i.e., $x_{0}=0 \in \mathbb{R}^{n}$. If this happens for all $k_{0} \in \mathbb{Z}$, (13) is simply called completely state observable.

\subsection{Property comparison}

We start by comparing the behavioral definition of reconstructibility with the classical definition given by Definition 4.1. For this purpose, consider a behavior $\mathfrak{B}$ consisting of the set of $(x, u, y)$ trajectories of an $n$-dimensional linear state space model, with $n$ states, $m$ inputs and $p$ outputs described by (13). It follows, from Definition 4.1, that the state space system is (completely 
state) reconstructible if there exists $k_{0} \leqslant k_{1}$ such that, for all $x_{0}=x\left(k_{0}\right)$,

$$
\left\{u \equiv 0,\left.y(k)\right|_{k \in\left[k_{0}, k_{1}-1\right]} \equiv 0\right\} \Rightarrow\left\{x\left(k_{1}\right)=0\right\}
$$

Suppose now that

$$
\left.(u, y)\right|_{\left[k_{0},+\infty\right)} \equiv(0,0)
$$

Then, in particular,

$$
\left.(u, y)\right|_{\left[k_{0}, k_{1}-1\right]} \equiv(0,0)
$$

and hence $x\left(k_{1}\right)=0$. Moreover, once $u \equiv 0$, the condition $x\left(k_{1}\right)=0$ clearly implies that

$$
\left.x(k)\right|_{\left[k_{1},+\infty\right)} \equiv 0,
$$

and thus

$$
\left\{\left.(u, y)\right|_{\left[k_{0},+\infty\right)} \equiv(0,0)\right\} \Rightarrow\left\{\left.x\right|_{\left[k_{1},+\infty\right)} \equiv 0\right\}
$$

That is, $x$ is behaviorally $\delta$-reconstructible from $(u, y)$, with $\delta=k_{1}-k_{0}$, as defined in the behavioral framework (cf (6)).

In order to see that the opposite implication also holds, assume that the state $x$ is reconstructible from $(u, y)$, in the behavioral sense. Furthermore, suppose that the state space system (13) is (classically) unreconstructible. Then, there exists a trajectory with $x\left(k_{0}\right)=x_{0}$ such that

$$
\forall \delta \geqslant 0,\left.(u, y)\right|_{\left[k_{0}, k_{0}+\delta-1\right]} \equiv(0,0) \quad \text { but } \quad x\left(k_{0}+\delta\right) \neq 0 .
$$

Observe that $x_{0} \neq 0$ because otherwise we have that

$$
\left\{x_{0}=0,\left.(u, y)\right|_{\left[k_{0}, k_{0}+\delta-1\right]} \equiv(0,0)\right\} \Rightarrow x\left(k_{0}+\delta\right)=0 .
$$

Furthermore, $x_{0}$ is (classically) unobservable which implies that if $u(k)=0$ for $k \geqslant k_{0}$, then $y(k)=0$ for $k \geqslant k_{0}$. Thus,

$$
\left.(u, y)\right|_{\left[k_{0},+\infty\right)} \equiv(0,0)
$$

and, therefore, there exists a trajectory $(x, u, y)$ such that

$$
\left.(u, y)\right|_{\left[k_{0},+\infty\right)} \equiv(0,0) \quad \text { and }\left.\quad x\right|_{\left[k_{0}+\delta,+\infty\right)} \neq 0
$$

as $x\left(k_{0}+\delta\right) \neq 0$, meaning that that state space system is not behaviorally reconstructible. Hence, it must be (classically) reconstructible.

Thus, this reasoning allows us to state the following result. 
Theorem 4.3: The state space system (13) is completely state reconstructible in the classical sense (Def.4.1) if and only if $x$ is reconstructible from $(u, y)$ in the behavioral sense (Def.3.1). $\diamond$

\subsection{Reconstructibility test}

Consider now the time-invariant version of the state space system (13) as a behavioral system $\mathfrak{B}$ whose variable is partitioned as $(w, v)$, with

$$
w:=\left[\begin{array}{l}
u \\
y
\end{array}\right] \text { and } \quad v:=x
$$

This leads to the following representation, in terms of $w$ and $v$,

$$
\left[\begin{array}{c}
\sigma I_{n}-A \\
C
\end{array}\right] x=\left[\begin{array}{cc}
B & 0 \\
-D & I_{p}
\end{array}\right] w
$$

and clearly, by Theorem 3.4, $x$ is reconstructible from $w$, in the behavioral sense, if and only if

$$
\operatorname{rank}\left[\begin{array}{c}
\lambda I_{n}-A \\
C
\end{array}\right]=n, \quad \forall \lambda \in \mathbb{C} \backslash\{0\},
$$

which coincides with the (classical) complete state reconstructibility rank condition, see (Urbano 1987, Hautus 1969, Kailath 1980, Kučera 1991).

Contrary to what happens with reconstructibility, the characterization of forward-observability for state space systems over $\mathbb{Z}$ does not coincide with the (classical) complete state observability rank condition, see (Urbano 1987, Hautus 1969, Kailath 1980, Kučera 1991). This is illustrated in the following example.

Example 4.4 Consider the state space system with no inputs, state $x=\left[\begin{array}{ll}x_{1} & x_{2}\end{array}\right]^{T}$ and output $y$, described by

$$
\left\{\begin{array}{r}
(\sigma x)(k)=A x(k) \\
y(k)=C x(k)
\end{array} \quad k \in \mathbb{Z}\right.
$$

with

$$
A=\left[\begin{array}{ll}
0 & 0 \\
0 & 1
\end{array}\right], \quad C=\left[\begin{array}{ll}
0 & 1
\end{array}\right]
$$

It turns out that the state $x$ is forward-observable from the output $y$, since the system trajectories satisfy $x_{1}=0$ and $x_{2}=y$. However,

$$
\left[\begin{array}{c}
\lambda I_{2}-A \\
C
\end{array}\right]
$$

has a rank drop for $\lambda=0$. Nevertheless, the description

$$
\underbrace{\left[\begin{array}{cc}
1 & 0 \\
0 & \sigma-1 \\
0 & 1
\end{array}\right]}_{\widetilde{R}_{2}(\sigma)} x=\left[\begin{array}{l}
0 \\
0 \\
1
\end{array}\right] y
$$


is such that

$$
\operatorname{rank} \widetilde{R}_{2}(\lambda)=2, \quad \forall \lambda \in \mathbb{C},
$$

it satisfies the condition of Theorem 3.4-ii).

If, instead of the time-invariant scenario, we now consider the $P$-periodic case, that is, if we take the matrices $A(k), B(k), C(k)$ and $D(k)$, in the state space system (13), to be periodic in $k$ with period $P \in \mathbb{N}$, then, by choosing the same variable partition as the one chosen in (14), we are led to the following representation, in terms of $w$ and $v$,

$$
\left(R_{t}\left(\sigma, \sigma^{-1}\right) w\right)(t+P k)=\left(M_{t}\left(\sigma, \sigma^{-1}\right) v\right)(t+P k), t=0, \ldots, P-1, k \in \mathbb{Z},
$$

with

$$
R_{t}\left(\xi, \xi^{-1}\right)=\left[\begin{array}{cc}
B(t) & 0 \\
-D(t) & I_{p}
\end{array}\right] \quad \text { and } \quad M_{t}\left(\xi, \xi^{-1}\right)=\left[\begin{array}{c}
\xi I_{n}-A(t) \\
C(t)
\end{array}\right]
$$

or still

$$
\left(R\left(\sigma, \sigma^{-1}\right) w\right)(P k)=\left(M\left(\sigma, \sigma^{-1}\right) v\right)(P k), k \in \mathbb{Z},
$$

with

$$
R\left(\xi, \xi^{-1}\right)=\left[\begin{array}{cc}
B(0) & 0 \\
-D(0) & I_{p} \\
\hline \xi B(1) & 0 \\
-\xi D(1) & \xi I_{p} \\
\hline \vdots & \vdots \\
\hline \xi^{P-1} B(P-1) & 0 \\
-\xi^{P-1} D(P-1) & \xi^{P-1} I_{p}
\end{array}\right] \quad \text { and } \quad M\left(\xi, \xi^{-1}\right)=\left[\begin{array}{c}
\xi I_{n}-A(0) \\
C(0) \\
\frac{\xi\left(\xi I_{n}-A(1)\right)}{\xi C(1)} \\
\vdots \\
\frac{\xi^{P-1}\left(\xi I_{n}-A(P-1)\right)}{\xi^{P-1} C(P-1)}
\end{array}\right] .
$$

The lifted behavior $L \mathfrak{B}$ corresponding to $\mathfrak{B}$ is described by

$$
\left(R^{L}\left(\sigma, \sigma^{-1}\right)(L w)\right)(k)=\left(M^{L}\left(\sigma, \sigma^{-1}\right)(L v)\right)(k), \quad k \in \mathbb{Z},
$$

where $M^{L}\left(\xi, \xi^{-1}\right) \in \mathbb{R}^{(n+p) P \times n P}\left[\xi, \xi^{-1}\right]$ is equal to

$$
\left[\begin{array}{c|ccc}
-A(0) & I_{n} & \cdots & 0 \\
C(0) & 0 & \cdots & 0 \\
0 & -A(1) & \cdots & 0 \\
0 & C(1) & \cdots & 0 \\
\vdots & \vdots & \ddots & \vdots \\
0 & 0 & \ddots & I_{n} \\
0 & 0 & \cdots & 0 \\
\xi I_{n} & 0 & \cdots & -A(P-1) \\
0 & 0 & \cdots & C(P-1)
\end{array}\right]
$$


By conveniently rearranging some block-rows in the matrix $M^{L}\left(\xi, \xi^{-1}\right)$ we obtain the matrix

$\left[\begin{array}{ccccccc}-A(0) & I_{n} & 0 & 0 & \cdots & 0 & 0 \\ 0 & -A(1) & I_{n} & 0 & \cdots & 0 & 0 \\ \vdots & \vdots & \vdots & \vdots & \ddots & \vdots & \vdots \\ 0 & 0 & 0 & 0 & \cdots & -A(P-2) & I_{n} \\ \hline \xi I_{n} & 0 & \cdots & \cdots & \cdots & 0 & -A(P-1) \\ C(0) & 0 & \cdots & \cdots & \cdots & \cdots & 0 \\ 0 & C(1) & 0 & \cdots & \cdots & \cdots & 0 \\ \vdots & 0 & \ddots & & & & \vdots \\ \vdots & \vdots & & \ddots & & & \vdots \\ \vdots & \vdots & & & \ddots & & \vdots \\ \vdots & \vdots & & & & \ddots & 0 \\ 0 & 0 & \cdots & \cdots & \cdots & 0 & C(P-1)\end{array}\right]$,

which we denote by $\widetilde{M^{L}}\left(\xi, \xi^{-1}\right)$.

Now, consecutively performing the $P-1$ block-column operations

$$
C_{j} \leftarrow C_{j}+C_{j+1} A(j-1), \quad j=P-1, \ldots, 1,
$$

(where $C_{j}$ is the $j^{\text {th }}$ block-column of matrix $\widetilde{M^{L}}\left(\xi, \xi^{-1}\right)$ ), i.e., taking in the first step $j=P-1$ and in the $(P-1)^{\text {th }}$ step $j=1$, we obtain the following matrix

$$
\widehat{M^{L}}\left(\xi, \xi^{-1}\right)=\left[\begin{array}{c|c}
0 & I_{(P-1) n} \\
\hline \widehat{M_{1}^{L}}\left(\xi, \xi^{-1}\right) & \widehat{M_{2}^{L}}\left(\xi, \xi^{-1}\right)
\end{array}\right],
$$

with

$$
\widehat{M_{1}^{L}}\left(\xi, \xi^{-1}\right)=\left[\begin{array}{c}
\xi I_{n}-A(P-1) \cdots A(0) \\
C(0) \\
C(1) A(0) \\
C(2) A(1) A(0) \\
\vdots \\
C(P-1) A(P-2) \cdots A(0)
\end{array}\right]
$$

Taking Theorem 3.6 into account we conclude that $\mathfrak{B}$ is reconstructible with respect to $x$ if and only if

$$
\operatorname{rank} M^{L}\left(\lambda, \lambda^{-1}\right)=n P, \quad \forall \lambda \in \mathbb{C} \backslash\{0\}
$$

Clearly the rank of the original $M^{L}$ matrix coincides with the rank of matrix (16) and, therefore,

$$
\forall \lambda \in \mathbb{C}, \quad \operatorname{rank} M^{L}\left(\lambda, \lambda^{-1}\right)=n(P-1)+\operatorname{rank} \widehat{M_{1}^{L}}\left(\lambda, \lambda^{-1}\right)
$$


where

$$
\operatorname{rank} \widehat{M_{1}^{L}}\left(\lambda, \lambda^{-1}\right)=\operatorname{rank}\left[\begin{array}{c}
\lambda I_{n}-A(P-1) \cdots A(0) \\
C(0) \\
C(1) A(0) \\
C(2) A(1) A(0) \\
\vdots \\
C(P-1) A(P-2) \cdots A(0)
\end{array}\right]
$$

Therefore $\mathfrak{B}$ is behaviorally reconstructible with respect to $x$ if and only if

$$
\operatorname{rank}\left[\begin{array}{c}
\lambda I_{n}-A(P-1) \cdots A(0) \\
C(0) \\
C(1) A(0) \\
C(2) A(1) A(0) \\
\vdots \\
C(P-1) A(P-2) \cdots A(0)
\end{array}\right]=n, \forall \lambda \in \mathbb{C} \backslash\{0\} .
$$

Remark 3: As expected, it can be shown that this rank condition is equivalent to the rank conditions obtained in (Urbano 1987, Hernández and Urbano 1987), for reconstructibility of periodic state space systems, based on a different time-invariant formulation.

\section{Conclusion}

In this paper, we have introduced and characterized the properties of reconstructibility and forward-observability for systems over $\mathbb{Z}$. A comparison was made with the existing results in the behavioral setting, for the time-invariant case. It turned out that our reconstructibility property is equivalent to Willems-observability. Moreover, for the case of state space systems (over $\mathbb{Z}$ ), the characterization of reconstructibility coincides with the well-known reconstructibility condition for systems over $\mathbb{Z}_{+}$. However the characterization of forward-observability is different from the observability condition for state space systems over $\mathbb{Z}_{+}$. A reconstructibility test has been obtained for period behaviors and a property comparison has been achieved within the classical state space framework, allowing us to conclude the equivalence between behavioral reconstructibility and state reconstructibility. Our results not only provide an overview of the relationship between different behavioral properties, but also establish a bridge between the behavioral and the classical state space settings.

\section{Acknowledgment}

The research of José C. Aleixo and Paula Rocha was partially supported by FEDER funds through COMPETE-Operational Programme Factors of Competitiveness ("Programa Operacional Factores de Competitividade") and by Portuguese funds through the Center for Research and Development in Mathematics and Applications (University of Aveiro) and the Portuguese Foundation for Science and Technology ("FCT-Fundação para a Ciência e a Tecnologia"), within project PEst-C/MAT/UI4106/2011 with COMPETE number FCOMP-01-0124-FEDER-022690. 


\section{References}

Aleixo, J.C., de Lima, T.P., and Rocha, P. (2008), "Comparison of the property of state-reconstructibility with behavioral reconstructibility for periodic systems," in Proceedings of the 8th Portuguese Conference on Automatic Control (Controlo 2008), Vila Real de Trás-os-Montes e Alto Douro, Portugal, 21-23 July 2008, pp. 846-851.

Aleixo, J.C., Polderman, J.W., and Rocha, P. (2007), "Representations and structural properties of periodic systems," Automatica J. IFAC, 43, 1921-1931.

Aleixo, J.C., and Rocha, P. (2007), "Reconstructibility and forward-observability of behaviors over $\mathbb{Z}$," in Proceedings of the 3rd IFAC Symposium on System, Structure and Control (SSSC 2007), Foz do Iguaçu, Brazil, 17-19 October 2007, Vol. 3.

Hautus, M.L.J. (1969), "Controllability and observability conditions of linear autonomous systems," Nederl. Akad. Wetensch. Proc. Ser. A, 72, 443-448.

Hernández, V., and Urbano, A. (1987), "Pole-assignment problem for discrete-time linear periodic systems," Internat. J. Control, 46, 687-697.

Kailath, T. (1980), Linear systems, Englewood Cliffs, NJ: Prentice-Hall, Inc.

Khargonekar, P.P., Poolla, K., and Tannenbaum, A. (1985), "Robust control of linear time-invariant plants using periodic compensation," IEEE Trans. Automat. Control, 30, 1088-1096.

Kučera, V. (1991), Analysis and design of discrete linear control systems, Englewood Cliffs, NJ: Prentice Hall International.

Kuijper, M., and Willems, J.C. (1997), "A behavioral framework for periodically time-varying systems," in Proceedings of the 36th IEEE Conference on Decision 83 Control - CDC'97 (San Diego, California USA, 1997), San Diego, California USA, 10-12 Dec. 1997, Vol. 3, pp. 2013-2016.

Lomadze, V., Ravi, M.S., Rosenthal, J., and Schumacher, J.M. (1998), "A Behavioral Approach to Singular Systems," Acta Applicandae Mathematicae, 54, 331-344.

Polderman, J.W., and Willems, J.C. (1998), Vol. 26 of Texts in Applied Mathematics, Introduction to mathematical systems theory: A Behavioral Approach, New York: Springer-Verlag.

Urbano, A. (1987), "Estabilidad y control óptimo de sistemas lineales periódicos discretos," Ph.D. dissertation, University of Valencia, Spain.

Valcher, M.E., and Willems, J.C. (1999b), "Dead beat observer synthesis," Systems Control Lett., 37, $285-292$.

Valcher, M.E., and Willems, J.C. (1999a), "Observer synthesis in the behavioral approach," IEEE Trans. Automat. Control, 44, 2297-2307.

Willems, J.C. (1989), Vol. 2 of Dynam. Report. Ser. Dynam. Systems Appl., "Models for dynamics," Dynamics reported, Chichester: Wiley, pp. 171-269.

Willems, J.C. (1991), "Paradigms and puzzles in the theory of dynamical systems," IEEE Trans. Automat. Control, 36, 259-294. 